\title{
Dual ordered structures of binary relations
}

\author{
V P Tsvetov ${ }^{1}$ \\ ${ }^{1}$ Samara National Research University, Moskovskoe shosse 34, Samara, Russia, 443086
}

\begin{abstract}
The theory of ordered structures like a (lattice) ordered semigroups is applied to graphs and automatons as well as to coding, programming and artificial intelligence. In this paper an algebraic structure on an underlying set of binary relations is considered. The structure includes the operations of Boolean algebra, inverse and composition. It is defined a dual semigroup to the binary relations ordered semigroup, and then the general properties of dual operations are studied.
\end{abstract}

\section{Introduction}

Abstract theory of algebraic structures (sometimes called universal algebra) forms the basis for various applications [1-8]. Semigroups and lattices are the simplest structures but not the least ones.

Let's recall some definitions:

The semigroup is a set with single binary operation * satisfying associative low. A semigroup with neutral (identity) element is called a monoid;

The semiring is a set with couple of binary operations - addition and multiplication - satisfying associative lows. There are neutral elements for both of them and addition is commutative. Also multiplication distributes over addition and multiplication by zero annihilates semiring;

The lattice (as an algebraic structure) is a set with pair of binary operations - join and meet satisfying associative lows, commutative lows, and absorption lows. A distributive lattice is a lattice in which the operations of join and meet distribute over each other. A bounded lattice is a lattice with neutral elements. The lattice's bottom is a neutral element for the join operation and the lattice's top is a neutral element for the meet operation;

The lattice (as a poset) is a partial ordered set such that each finite-elements subset has supremum (join) and infimum (meet). A bounded lattice is a lattice with bottom and top elements;

The ordered semigroup is a semigroup together with a partial order $\prec$ that is compatible with the semigroup operation i.e. $\forall u, v, w u \prec v \rightarrow w * u \prec w \circ v \wedge u * w \prec v * w$. The bounded semigroup is an ordered semigroup with bottom and top elements.

It's well known that any ordered semigroup is isomorphic to a subsemigroup of binary relations ordered by subset relation. In this paper we deal with a left composition of binary relation as a semigroup operation, i.e. we set

$$
R_{1} \circ R_{2}=\left\{\left(u_{1}, u_{2}\right) \mid \exists u_{3}\left(u_{1}, u_{3}\right) \in R_{1} \wedge\left(u_{3}, u_{2}\right) \in R_{2}\right\}
$$

At first, we denote a universe as $U$ and consider a power set of Cartesian square $2^{U \times U}$ as a collection of binary relations on $U$. The traditional approach to studying binary relations leads to ordered semigroup $S_{\mathrm{R}}=\left\langle 2^{U \times U},(\circ, \subseteq)\right\rangle$ and bounded distributive lattice $L_{\mathrm{R}}=\left\langle 2^{U \times U},(\cup, \cap)\right\rangle$. In this way we don't take into account a complement operation 


$$
\bar{R}=\left\{\left(u_{1}, u_{2}\right) \mid\left(u_{1}, u_{2}\right) \notin R\right\}
$$

However, it's very convenient to use a complement element. For example, we can write the trichotomy low for relation $R$ in several forms. First, we can write it as in equation (3)

$$
I_{\mathrm{R}} \cup R \cup R^{-1}=1_{\mathrm{R}}=U \times U=\bar{\varnothing}=\overline{0_{\mathrm{R}}}
$$

Then, we can rewrite it in alternative form as antisymmetric low for the complement $\bar{R}$ as in equation (4)

$$
\bar{R} \cap \bar{R}^{-1} \subseteq I_{\mathrm{R}}=\{(u, u) \mid u \in U\}
$$

In this case and below we use the notations $1_{\mathrm{R}}, 0_{\mathrm{R}}$ and $I_{\mathrm{R}}$ for complete relation, empty relation and identity relation respectively. Note that $1_{R}$ and $0_{R}$ are top and bottom elements for lattice $L_{R}$. Also we denote the inverse relation of $R$ as

$$
R^{-1}=\left\{\left(u_{2}, u_{1}\right) \mid\left(u_{1}, u_{2}\right) \in R\right\}
$$

\section{Algebraic structure $h_{\mathrm{R}}$}

Let's consider an algebraic structure $h_{\mathrm{R}}=\left\langle 2^{U \times U},\left(\cup, \cap, \circ,{ }^{-1}, \subseteq, 0_{\mathrm{R}}, 1_{\mathrm{R}}, I_{\mathrm{R}}\right)\right\rangle$. It's easy to prove properties (6)-(41):

$$
\begin{gathered}
R_{1} \cup\left(R_{2} \cup R_{3}\right)=\left(R_{1} \cup R_{2}\right) \cup R_{3} \\
R_{1} \cap\left(R_{2} \cap R_{3}\right)=\left(R_{1} \cap R_{2}\right) \cap R_{3} \\
R_{1} \circ\left(R_{2} \circ R_{3}\right)=\left(R_{1} \circ R_{2}\right) \circ R_{3} \\
R_{1} \cup R_{2}=R_{2} \cup R_{1} \\
R_{1} \cap R_{2}=R_{2} \cap R_{1} \\
0_{\mathrm{R}} \cup R=R \\
1_{\mathrm{R}} \cap R=R \\
I_{\mathrm{R}} \circ R=R \circ I_{\mathrm{R}}=R \\
1_{\mathrm{R}} \cup R=1_{\mathrm{R}} \\
0_{\mathrm{R}} \cap R=0_{\mathrm{R}} \\
0_{\mathrm{R}} \circ R=R \circ 0_{\mathrm{R}}=0_{\mathrm{R}} \\
1_{\mathrm{R}} \circ 1_{\mathrm{R}}=1_{\mathrm{R}} \\
R_{1} \cup\left(R_{2} \cap R_{3}\right)=\left(R_{1} \cup R_{2}\right) \cap\left(R_{1} \cup R_{3}\right) \\
R_{1} \cap\left(R_{2} \cup R_{3}\right)=\left(R_{1} \cap R_{2}\right) \cup\left(R_{1} \cap R_{3}\right) \\
R_{1} \circ\left(R_{2} \cup R_{3}\right)=\left(R_{1} \circ R_{2}\right) \cup\left(R_{1} \circ R_{3}\right) \\
\left(R_{2} \cup R_{3}\right) \circ R_{1}=\left(R_{2} \circ R_{1}\right) \cup\left(R_{3} \circ R_{1}\right) \\
R_{1} \circ\left(R_{2} \cap R_{3}\right) \subseteq\left(R_{1} \circ R_{2}\right) \cap\left(R_{1} \circ R_{3}\right) \\
\left(R_{2} \cap R_{3}\right) \circ R_{1} \subseteq\left(R_{2} \circ R_{1}\right) \cap\left(R_{3} \circ R_{1}\right) \\
R_{1} \cup\left(R_{1} \cap R_{2}\right)=R_{1} \\
R_{1} \cap\left(R_{1} \cup R_{2}\right)=R_{1} \\
R \cup R=R \\
R \cap R=R \\
\overline{\bar{R}}=R \\
\overline{1_{\mathrm{R}}}=0_{\mathrm{R}} \\
\overline{0_{\mathrm{R}}}=1_{\mathrm{R}} \\
\end{gathered}
$$




$$
\begin{gathered}
\overline{R_{1} \cup R_{2}}=\bar{R}_{1} \cap \bar{R}_{2} \\
\overline{R_{1} \cap R_{2}}=\bar{R}_{1} \cup \bar{R}_{2} \\
\left(R_{1} \cup R_{2}\right)^{-1}=R_{1}^{-1} \cup R_{2}^{-1} \\
\left(R_{1} \cap R_{2}\right)^{-1}=R_{1}^{-1} \cap R_{2}^{-1} \\
\left(R_{1} \circ R_{2}\right)^{-1}=R_{2}^{-1} \circ R_{1}^{-1} \\
\overline{R^{-1}}=\bar{R}^{-1} \\
R_{1} \subseteq R_{2} \Leftrightarrow R_{1} \cup R_{2}=R_{2} \Leftrightarrow R_{1} \cap R_{2}=R_{1} \\
R_{1} \subseteq R_{2} \Rightarrow R_{1} \cup R_{3} \subseteq R_{2} \cup R_{3} \\
R_{1} \subseteq R_{2} \Rightarrow R_{1} \cap R_{3} \subseteq R_{2} \cap R_{3} \\
R_{1} \subseteq R_{2} \Rightarrow R_{1} \circ R_{3} \subseteq R_{2} \circ R_{3} \wedge R_{3} \circ R_{1} \subseteq R_{3} \circ R_{2} \\
R_{1} \subseteq R_{2} \Leftrightarrow \bar{R}_{1} \supseteq \bar{R}_{2}
\end{gathered}
$$

The typical algebraic structures we can obtain by restriction of structure $h_{R(U \times U)}$ are as follows:

The bounded lattices of binary relations $L O_{\mathrm{R}}^{1}=\left\langle 2^{U \times U},\left(\subseteq, 0_{\mathrm{R}}, 1_{\mathrm{R}}\right)\right\rangle$ and $L O_{\mathrm{R}}^{2}=\left\langle 2^{U \times U},\left(\supseteq, 1_{\mathrm{R}}, 0_{\mathrm{R}}\right)\right\rangle$;

The bounded monoids of binary relations $M_{\mathrm{R}}^{1}=\left\langle 2^{U \times U},\left(\cup, \subseteq, 0_{\mathrm{R}}, 1_{\mathrm{R}}\right)\right\rangle, M_{\mathrm{R}}^{2}=\left\langle 2^{U \times U},\left(\cap, \subseteq, 0_{\mathrm{R}}, 1_{\mathrm{R}}\right)\right\rangle$, $M_{\mathrm{R}}^{3}=\left\langle 2^{U \times U},\left(\cup, \supseteq, 1_{\mathrm{R}}, 0_{\mathrm{R}}\right)\right\rangle, M_{\mathrm{R}}^{4}=\left\langle 2^{U \times U},\left(\cap, \supseteq, 1_{\mathrm{R}}, 0_{\mathrm{R}}\right)\right\rangle$ and $M_{\mathrm{R}}=\left\langle 2^{U \times U},\left(\circ, \subseteq, I_{\mathrm{R}}, 0_{\mathrm{R}}, 1_{\mathrm{R}}\right)\right\rangle$;

The bounded semirings (with multiplicative identity) of binary relations $S R_{\mathrm{R}}^{1}=\left\langle 2^{U \times U},\left(\cup, \cap, \subseteq, 0_{\mathrm{R}}, 1_{\mathrm{R}}\right)\right\rangle, S R_{\mathrm{R}}^{2}=\left\langle 2^{U \times U},\left(\cap, \cup, \supseteq, 1_{\mathrm{R}}, 0_{\mathrm{R}}\right)\right\rangle$, and $S R_{\mathrm{R}}=\left\langle 2^{U \times U},\left(\cup, \circ, \subseteq, 0_{\mathrm{R}}, I_{\mathrm{R}}\right)\right\rangle$;

The Boolean algebras of binary relations $B_{\mathrm{R}}^{1}=\left\langle 2^{U \times U},\left(\cup, \cap,{ }^{-}, 0_{\mathrm{R}}, 1_{\mathrm{R}}\right)\right\rangle \quad$ and $B_{\mathrm{R}}^{2}=\left\langle 2^{U \times U},\left(\cap, \cup^{-}, 1_{\mathrm{R}}, 0_{\mathrm{R}}\right)\right\rangle$.

\section{Dual semigroup to $S_{\mathrm{R}}$}

Let's consider a Boolean isomorphism $F(R)=\bar{R}$ from $B_{\mathrm{R}}^{1}$ onto $B_{\mathrm{R}}^{2}$. We define a binary operation • in accordance with duality principle $F\left(R_{1} \bullet R_{2}\right)=F\left(R_{1}\right) \circ F\left(R_{2}\right)$, i.e. we set

$$
R_{1} \bullet R_{2}=\overline{\overline{R_{1}} \circ \overline{R_{2}}}=\left\{\left(u_{1}, u_{2}\right) \mid \forall u_{3}\left(u_{1}, u_{3}\right) \in R_{1} \vee\left(u_{3}, u_{2}\right) \in R_{2}\right\} .
$$

Note that $F\left(0_{\mathrm{R}}\right)=1_{\mathrm{R}}, F\left(1_{\mathrm{R}}\right)=0_{\mathrm{R}}, F\left(I_{\mathrm{R}}\right)=\overline{I_{\mathrm{R}}}$ and moreover

$$
\begin{gathered}
R_{1} \circ R_{2}=\overline{\overline{R_{1}} \cdot \overline{R_{2}}}=\left\{\left(u_{1}, u_{2}\right) \mid \exists u_{3}\left(u_{1}, u_{3}\right) \in R_{1} \wedge\left(u_{3}, u_{2}\right) \in R_{2}\right\} \\
R_{1} \cdot\left(R_{2} \cdot R_{3}\right)=\left(R_{1} \cdot R_{2}\right) \cdot R_{3} \\
\overline{I_{\mathrm{R}}} \cdot R=R \cdot \overline{I_{\mathrm{R}}}=R \\
1_{\mathrm{R}} \cdot R=R \cdot 1_{\mathrm{R}}=1_{\mathrm{R}} \\
0_{\mathrm{R}} \cdot 0_{\mathrm{R}}=0_{\mathrm{R}} \\
R_{1} \cdot\left(R_{2} \cap R_{3}\right)=\left(R_{1} \cdot R_{2}\right) \cap\left(R_{1} \cdot R_{3}\right) \\
\left(R_{2} \cap R_{3}\right) \cdot R_{1}=\left(R_{2} \cdot R_{1}\right) \cap\left(R_{3} \cdot R_{1}\right) \\
R_{1} \cdot\left(R_{2} \cup R_{3}\right) \supseteq\left(R_{1} \cdot R_{2}\right) \cup\left(R_{1} \cdot R_{3}\right) \\
\left(R_{2} \cup R_{3}\right) \cdot R_{1} \supseteq\left(R_{2} \cdot R_{1}\right) \cup\left(R_{3} \cdot R_{1}\right) \\
\left(R_{1} \cdot R_{2}\right)^{-1}=R_{2}^{-1} \cdot R_{1}^{-1} \\
R_{1} \supseteq R_{2} \Rightarrow R_{1} \cdot R_{3} \supseteq R_{2} \cdot R_{3} \wedge R_{3} \cdot R_{1} \supseteq R_{3} \cdot R_{2}
\end{gathered}
$$


By our construction semigroup $S_{\mathrm{R}}$ is isomorphic to semigroup $\bar{S}_{\mathrm{R}}=\left\langle 2^{U \times U},(\cdot, \supseteq)\right\rangle$ as well as monoid $M_{\mathrm{R}}$ and semiring $S R_{\mathrm{R}}$ are isomorphic to $\bar{M}_{\mathrm{R}}=\left\langle 2^{U \times U},\left(\cdot, \supseteq, \bar{I}_{\mathrm{R}}, 1_{\mathrm{R}}, 0_{\mathrm{R}}\right)\right\rangle$ and $\overline{S R}_{\mathrm{R}}=\left\langle 2^{U \times U},\left(\cap, \bullet, \supseteq, 1_{\mathrm{R}}, \overline{I_{\mathrm{R}}}\right)\right\rangle$ respectively. In such cases, we'll say that the algebraic structures are dual.

Now we use the previous definitions to argue the following logical consequences:

$$
\begin{gathered}
u_{1} R_{1} \circ\left(R_{2} \bullet R_{3}\right) u_{2} \Leftrightarrow \exists u_{3} u_{1} R_{1} u_{3} \wedge u_{3}\left(R_{2} \bullet R_{3}\right) u_{2} \Leftrightarrow \exists u_{3} u_{1} R_{1} u_{3} \wedge\left(\forall u_{4} u_{3} R_{2} u_{4} \vee u_{4} R_{3} u_{2}\right) \\
\Leftrightarrow \exists u_{3} \forall u_{4} u_{1} R_{1} u_{3} \wedge\left(u_{3} R_{2} u_{4} \vee u_{4} R_{3} u_{2}\right) \Leftrightarrow \exists u_{3} \forall u_{4}\left(u_{1} R_{1} u_{3} \wedge u_{3} R_{2} u_{4}\right) \vee\left(u_{1} R_{1} u_{3} \wedge u_{4} R_{3} u_{2}\right) \\
\Rightarrow \forall u_{4} \exists u_{3}\left(u_{1} R_{1} u_{3} \wedge u_{3} R_{2} u_{4}\right) \vee\left(u_{1} R_{1} u_{3} \wedge u_{4} R_{3} u_{2}\right) \Leftrightarrow \forall u_{4}\left(\exists u_{3} u_{1} R_{1} u_{3} \wedge u_{3} R_{2} u_{4}\right) \vee\left(\exists u_{3} u_{1} R_{1} u_{3} \wedge u_{4} R_{3} u_{2}\right) \\
\Leftrightarrow \forall u_{4} u_{1} R_{1} \circ R_{2} u_{4} \vee\left(\exists u_{3} u_{1} R_{1} u_{3} \wedge u_{4} R_{3} u_{2}\right) \Leftrightarrow \forall u_{4}\left(u_{1} R_{1} \circ R_{2} u_{4} \vee u_{4} R_{3} u_{2}\right) \wedge\left(u_{1} R_{1} \circ R_{2} u_{4} \vee \exists u_{3} u_{1} R_{1} u_{3}\right) \\
\Leftrightarrow\left(\forall u_{4} u_{1} R_{1} \circ R_{2} u_{4} \vee u_{4} R_{3} u_{2}\right) \wedge\left(\forall u_{4} u_{1} R_{1} \circ R_{2} u_{4} \vee \exists u_{3} u_{1} R_{1} u_{3}\right) \\
\Leftrightarrow u_{1} R_{1} \circ\left(R_{2} \bullet R_{3}\right) u_{2} \wedge\left(\forall u_{4} u_{1} R_{1} \circ R_{2} u_{4} \vee \exists u_{3} u_{1} R_{1} u_{3}\right) \\
\Leftrightarrow u_{1} R_{1} \circ\left(R_{2} \bullet R_{3}\right) u_{2} \wedge\left(\left(\forall u_{4} \exists u_{3} u_{1} R_{1} \circ R_{2} u_{4}\right) \vee\left(\exists u_{3} u_{1} R_{1} u_{3}\right)\right) \Rightarrow u_{1} R_{1} \circ\left(R_{2} \bullet R_{3}\right) u_{2} \wedge\left(\exists u_{3} u_{1} R_{1} u_{3}\right) \\
\Leftrightarrow u_{1} R_{1} \circ\left(R_{2} \bullet R_{3}\right) u_{2} \wedge\left(u_{1} \in D_{R_{1}}\right)
\end{gathered}
$$

Let's denote a domain of $R_{1}$ as $D_{R_{1}}=\left\{u_{1} \mid \exists u_{3} u_{1} R_{1} u_{3}\right\} \subseteq U$ and then consider a binary relation $E\left(D_{R_{1}}, 1_{\mathrm{R}}\right)=\left\{\left(u_{1}, u_{2}\right) \mid u_{1} \in D_{R_{1}} \wedge u_{2} \in U\right\}=D_{R_{1}} \times U \subseteq 1_{\mathrm{R}}=U \times U$. Now we can write

$$
R_{1} \circ\left(R_{2} \cdot R_{3}\right) \subseteq\left(R_{1} \circ R_{2}\right) \cdot R_{3} \cap E\left(D_{R_{1}}, 1_{\mathrm{R}}\right)
$$

Note that

$u_{1} R_{1} \circ 1_{\mathrm{R}} u_{2} \Leftrightarrow \exists u_{3} u_{1} R_{1} u_{3} \wedge u_{3} 1_{\mathrm{R}} u_{2} \Leftrightarrow \exists u_{3} u_{1} R_{1} u_{3} \wedge u_{2} \in U \Leftrightarrow u_{1} \in D_{R_{1}} \wedge u_{2} \in U \Leftrightarrow u_{1} E\left(D_{R_{1}}, 1_{\mathrm{R}}\right) u_{2}$ and so we obtain

Similarly, we get

$$
R_{1} \circ\left(R_{2} \cdot R_{3}\right) \subseteq\left(R_{1} \circ R_{2}\right) \cdot R_{3} \cap R_{1} \circ 1_{\mathrm{R}}
$$

$$
\begin{aligned}
& \left(R_{2} \cdot R_{3}\right) \circ R_{1} \subseteq R_{2} \cdot\left(R_{3} \circ R_{1}\right) \cap 1_{\mathrm{R}} \circ R_{1} \\
& R_{1} \cdot\left(R_{2} \circ R_{3}\right) \supseteq\left(R_{1} \cdot R_{2}\right) \circ R_{3} \cup R_{1} \bullet 0_{\mathrm{R}} \\
& \left(R_{2} \circ R_{3}\right) \cdot R_{1} \supseteq R_{2} \circ\left(R_{3} \cdot R_{1}\right) \cup 0_{\mathrm{R}} \cdot R_{1}
\end{aligned}
$$

In the latter, we have taken into account the following equalities:

$$
\begin{aligned}
& E\left(1_{\mathrm{R}}, D_{R_{1}^{-1}}\right)=U \times D_{R_{1}^{-1}}=1_{\mathrm{R}} \circ R_{1} \\
& E\left(\overline{D_{\overline{R_{1}}}}, 1_{\mathrm{R}}\right)=\overline{D_{\overline{R_{1}}}} \times U=R_{1} \cdot 0_{\mathrm{R}} \\
& E\left(1_{\mathrm{R}}, \overline{D_{\bar{R}_{1}^{-1}}}\right)=U \times \overline{D_{\bar{R}_{1}^{-1}}}=0_{\mathrm{R}} \cdot R_{1}
\end{aligned}
$$

\section{Extension of algebraic structure $h_{\mathrm{R}}$}

At first, we denote $O_{\mathrm{R}}=\overline{I_{\mathrm{R}}}$ and then we consider $H_{\mathrm{R}}=\left\langle 2^{U \times U},\left(\cup, \cap, \bullet, \circ,{ }^{-},{ }^{-1}, \subseteq, 0_{\mathrm{R}}, 1_{\mathrm{R}}, O_{\mathrm{R}}, I_{\mathrm{R}}\right)\right\rangle$ as an extension of algebraic structure $h_{\mathrm{R}}$. It is clear that all of the properties (6)-(58) are true for structure $H_{\mathrm{R}}$.

Let's rewrite (57)-(58) in the form

$$
\begin{aligned}
& \left(R_{2} \cdot R_{3}\right) \circ R_{1} \cup R_{2} \cdot 0_{\mathrm{R}} \subseteq R_{2} \cdot\left(R_{3} \circ R_{1}\right) \\
& R_{1} \circ\left(R_{2} \cdot R_{3}\right) \cup 0_{\mathrm{R}} \cdot R_{3} \subseteq\left(R_{1} \circ R_{2}\right) \cdot R_{3}
\end{aligned}
$$

So we can rewrite (55)-(58) as: 


$$
\begin{aligned}
& R_{1} \circ\left(R_{2} \cdot R_{3}\right) \subseteq\left(R_{1} \circ R_{2}\right) \cdot R_{3} \cap R_{1} \circ 1_{\mathrm{R}} \\
& R_{1} \circ\left(R_{2} \cdot R_{3}\right) \cup 0_{\mathrm{R}} \cdot R_{3} \subseteq\left(R_{1} \circ R_{2}\right) \cdot R_{3} \\
& \left(R_{2} \cdot R_{3}\right) \circ R_{1} \subseteq R_{2} \cdot\left(R_{3} \circ R_{1}\right) \cap 1_{\mathrm{R}} \circ R_{1} \\
& \left(R_{2} \cdot R_{3}\right) \circ R_{1} \cup R_{2} \cdot 0_{\mathrm{R}} \subseteq R_{2} \cdot\left(R_{3} \circ R_{1}\right)
\end{aligned}
$$

Obviously, for all binary relations $R_{1}, R_{2}, R_{3} \in 2^{U \times U}$ we have

$$
\begin{aligned}
& R_{1} \circ\left(R_{2} \cdot R_{3}\right) \subseteq\left(R_{1} \circ R_{2}\right) \cdot R_{3} \\
& \left(R_{2} \bullet R_{3}\right) \circ R_{1} \subseteq R_{2} \cdot\left(R_{3} \circ R_{1}\right)
\end{aligned}
$$

This is immediate from the inclusions (59)-(62).

Properties like the (55)-(58), (63)-(64) we'll call the laws of semi-compatibility. Now we are interested in cases of compatibility (low) of dual operation with each other

$$
\begin{aligned}
& R_{1} \circ\left(R_{2} \cdot R_{3}\right)=\left(R_{1} \circ R_{2}\right) \cdot R_{3}=R_{1} \circ R_{2} \cdot R_{3} \\
& \left(R_{2} \cdot R_{3}\right) \circ R_{1}=R_{2} \bullet\left(R_{3} \circ R_{1}\right)=R_{2} \bullet R_{3} \circ R_{1}
\end{aligned}
$$

Note that we won't find algebraic substructures of $H_{\mathrm{R}}$ satisfying (65)-(66). Indeed, from (16), (17), (46), (47) we obtain

$$
\begin{aligned}
& 0_{R} \circ\left(0_{R} \bullet 1_{R}\right)=0_{R} \neq 1_{R}=\left(0_{R} \circ 0_{R}\right) \cdot 1_{R} \\
& 0_{R} \circ\left(1_{R} \bullet 1_{R}\right)=0_{R} \neq 1_{R}=\left(0_{R} \circ 1_{R}\right) \cdot 1_{R} \\
& \left(1_{R} \bullet 0_{R}\right) \circ 0_{R}=0_{R} \neq 1_{R}=1_{R} \cdot\left(0_{R} \circ 0_{R}\right) \\
& \left(1_{R} \bullet 1_{R}\right) \circ 0_{R}=0_{R} \neq 1_{R}=1_{R} \cdot\left(1_{R} \circ 0_{R}\right)
\end{aligned}
$$

Hence, we have to restrict structure $H_{\mathrm{R}}$ to find algebraic substructures satisfying (65)-(66) and we'll call them compatible (sub)structures. Let's consider $H_{\mathrm{R}}$ without $0_{\mathrm{R}}$ or $1_{\mathrm{R}}$.

We are studying the simplest cases of subsets of $2^{U \times U}$ as an underlying set for the operations from $H_{\mathrm{R}}$ below.

Let's denote the collection of (partial) functions from $U$ to $U$ as $U_{+1}^{U} \subseteq 2^{U \times U}$. It's easy that $\left\langle U_{+1}^{U},\left(\circ, \subseteq, I_{U \times U}, 0_{U \times U}\right)\right\rangle$ is a bounded below submonoid of $M_{\mathrm{R}}$.

We want to prove that $U_{+1}^{U}$ is closed under the dual operation $\bullet$.

At first, we suppose that $U$ contains only one element. In this case $U_{+1}^{U}=2^{U \times U}=\left\{I_{\mathrm{R}}, 0_{\mathrm{R}}\right\}$, where $I_{\mathrm{R}}$ is identity function and $0_{\mathrm{R}}$ is empty function. So $U_{+1}^{U}$ is closed under $\bullet$ because $2^{U \times U}$ is closed.

Let now $U$ contains more than one element. Suppose $R_{1}, R_{2} \in U_{+1}^{U}$, but $R_{1} \bullet R_{2} \notin U_{+1}^{U}$. Hence, there are $u_{1}, u_{2}, u_{3} \in U$ such that $u_{2} \neq u_{3}$ and $\left(\left(u_{1}, u\right) \in R_{1} \vee\left(u, u_{2}\right) \in R_{2}\right) \wedge\left(\left(u_{1}, u\right) \in R_{1} \vee\left(u, u_{3}\right) \in R_{2}\right)$ for all $u \in U$. However, $R_{1} \in U_{+1}^{U}$ and so there is no more than one $u_{0} \in U$ satisfying relation $\left(u_{1}, u_{0}\right) \in R_{1}$. Whence $u_{2} \neq u_{3} \wedge\left(u, u_{2}\right) \in R_{2} \wedge\left(u, u_{3}\right) \in R_{2}$ for all $u \in U \backslash\left\{u_{0}\right\} \neq \varnothing$. The latter is in contradiction with $R_{2} \in U_{+1}^{U}$.

The proof is complete.

Let's consider the scale of sets $U_{0}^{U} \subseteq U^{U} \subseteq U_{+1}^{U}$, where $U^{U}$ is a collection of total functions and $U_{0}^{U}$ is a collection of total bijections from $U$ to $U$.

We assume $U$ to be a two-element set and denote the cardinality of $U=\left\{u_{1}, u_{2}\right\}$ as $|U|$. Obviously, $\left|2^{U \times U}\right|=16,\left|U_{+1}^{U}\right|=9,\left|U^{U}\right|=4,\left|U_{0}^{U}\right|=|U|=2$. Note $1_{\mathrm{R}} \notin U_{+1}^{U}, 0_{\mathrm{R}} \in U_{+1}^{U}, 0_{\mathrm{R}} \notin U^{U}$.

We have simulated some interesting cases of algebraic substructures to check irregularities in (65)(66). Table 1 contains statistics on the incompatibility of dual operations. 
Table 1. A total amount of incompatibility of $\circ$ and $\bullet$.

\begin{tabular}{ccc}
\hline$R_{1}, R_{2}, R_{3} \in W$ & $R_{1} \circ\left(R_{2} \bullet R_{3}\right) \neq\left(R_{1} \circ R_{2}\right) \cdot R_{3}$ & $\left(R_{2} \bullet R_{3}\right) \circ R_{1} \neq R_{2} \bullet\left(R_{3} \circ R_{1}\right)$ \\
\hline$W=2^{U \times U} \backslash\left\{0_{\mathrm{R}}\right\}$ & 706 from 3375 & 706 from 3375 \\
$W=2^{U \times U} \backslash\left\{1_{\mathrm{R}}\right\}$ & 706 from 3375 & 706 from 3375 \\
$W=U_{+1}^{U}$ & 90 from 729 & 20 from 729 \\
$W=U^{U}$ & 0 from 64 & 0 from 64 \\
$W=U^{U} \cup\left\{0_{\mathrm{R}}\right\}$ & 10 from 75 & 4 from 75 \\
$W=U^{U} \cup\left\{1_{\mathrm{R}}\right\}$ & 4 from 75 & 10 from 75 \\
$W=U_{0}^{U}$ & 0 from 8 & 0 from 8 \\
$W=U_{0}^{U} \cup\left\{0_{\mathrm{R}}\right\}$ & 0 from 27 & 0 from 27 \\
$W=U_{0}^{U} \cup\left\{1_{\mathrm{R}}\right\}$ & 0 from 27 & 0 from 27
\end{tabular}

Cayley tables 2 and 3 describes the dual operations on the set $U_{0}^{U} \cup\left\{0_{\mathrm{R}}, 1_{\mathrm{R}}\right\}$.

Table 2. A Cayley table for $\circ$ on the set $U_{0}^{U} \cup\left\{0_{\mathrm{R}}, 1_{\mathrm{R}}\right\}$.

\begin{tabular}{ccccc}
\hline$\circ$ & $O_{\mathrm{R}}$ & $I_{\mathrm{R}}$ & $0_{\mathrm{R}}$ & $1_{\mathrm{R}}$ \\
\hline$O_{\mathrm{R}}$ & $I_{\mathrm{R}}$ & $O_{\mathrm{R}}$ & $0_{\mathrm{R}}$ & $1_{\mathrm{R}}$ \\
$I_{\mathrm{R}}$ & $O_{\mathrm{R}}$ & $I_{\mathrm{R}}$ & $0_{\mathrm{R}}$ & $1_{\mathrm{R}}$ \\
$0_{\mathrm{R}}$ & $0_{\mathrm{R}}$ & $0_{\mathrm{R}}$ & $0_{\mathrm{R}}$ & $0_{\mathrm{R}}$ \\
$1_{\mathrm{R}}$ & $1_{\mathrm{R}}$ & $1_{\mathrm{R}}$ & $0_{\mathrm{R}}$ & $1_{\mathrm{R}}$
\end{tabular}

Table 3. A Cayley table for - on the set $U_{0}^{U} \cup\left\{0_{\mathrm{R}}, 1_{\mathrm{R}}\right\}$.

\begin{tabular}{ccccc}
\hline$\bullet$ & $O_{\mathrm{R}}$ & $I_{\mathrm{R}}$ & $0_{\mathrm{R}}$ & $1_{\mathrm{R}}$ \\
\hline$O_{\mathrm{R}}$ & $O_{\mathrm{R}}$ & $I_{\mathrm{R}}$ & $0_{\mathrm{R}}$ & $1_{\mathrm{R}}$ \\
$I_{\mathrm{R}}$ & $I_{\mathrm{R}}$ & $O_{\mathrm{R}}$ & $0_{\mathrm{R}}$ & $1_{\mathrm{R}}$ \\
$0_{\mathrm{R}}$ & $0_{\mathrm{R}}$ & $0_{\mathrm{R}}$ & $0_{\mathrm{R}}$ & $1_{\mathrm{R}}$ \\
$1_{\mathrm{R}}$ & $1_{\mathrm{R}}$ & $1_{\mathrm{R}}$ & $1_{\mathrm{R}}$ & $1_{\mathrm{R}}$
\end{tabular}

The cases of incompatibility on the set $U_{0}^{U} \cup\left\{0_{\mathrm{R}}, 1_{\mathrm{R}}\right\}$ are listed below apart from (67)-(70). $R_{1} \circ\left(R_{2} \cdot R_{3}\right) \neq\left(R_{1} \circ R_{2}\right) \cdot R_{3}:$

$$
\begin{aligned}
1_{\mathrm{R}} \circ\left(I_{\mathrm{R}} \bullet 0_{\mathrm{R}}\right)=0_{\mathrm{R}} \neq 1_{\mathrm{R}}=\left(1_{\mathrm{R}} \circ I_{\mathrm{R}}\right) \cdot 0_{\mathrm{R}} \\
1_{\mathrm{R}} \circ\left(O_{\mathrm{R}} \bullet 0_{\mathrm{R}}\right)=0_{\mathrm{R}} \neq 1_{\mathrm{R}}=\left(1_{\mathrm{R}} \circ O_{\mathrm{R}}\right) \cdot 0_{\mathrm{R}} \\
0_{\mathrm{R}} \circ\left(I_{\mathrm{R}} \cdot 1_{\mathrm{R}}\right)=0_{\mathrm{R}} \neq 1_{\mathrm{R}}=\left(0_{\mathrm{R}} \circ I_{\mathrm{R}}\right) \cdot 1_{\mathrm{R}} \\
0_{\mathrm{R}} \circ\left(O_{\mathrm{R}} \bullet 1_{\mathrm{R}}\right)=0_{\mathrm{R}} \neq 1_{\mathrm{R}}=\left(0_{\mathrm{R}} \circ O_{\mathrm{R}}\right) \cdot 1_{\mathrm{R}}
\end{aligned}
$$

$\left(R_{2} \cdot R_{3}\right) \circ R_{1} \neq R_{2} \cdot\left(R_{3} \circ R_{1}\right):$

$$
\begin{gathered}
\left(0_{\mathrm{R}} \cdot I_{\mathrm{R}}\right) \circ 1_{\mathrm{R}}=0_{\mathrm{R}} \neq 1_{\mathrm{R}}=0_{\mathrm{R}} \cdot\left(I_{\mathrm{R}} \circ 1_{\mathrm{R}}\right) \\
\left(0_{\mathrm{R}} \cdot O_{\mathrm{R}}\right) \circ 1_{\mathrm{R}}=0_{\mathrm{R}} \neq 1_{\mathrm{R}}=0_{\mathrm{R}} \cdot\left(O_{\mathrm{R}} \circ 1_{\mathrm{R}}\right) \\
\left(1_{\mathrm{R}} \cdot I_{\mathrm{R}}\right) \circ 0_{\mathrm{R}}=0_{\mathrm{R}} \neq 1_{\mathrm{R}}=1_{\mathrm{R}} \cdot\left(I_{\mathrm{R}} \circ 0_{\mathrm{R}}\right) \\
\left(1_{\mathrm{R}} \cdot O_{\mathrm{R}}\right) \circ 0_{\mathrm{R}}=0_{\mathrm{R}} \neq 1_{\mathrm{R}}=1_{\mathrm{R}} \cdot\left(O_{\mathrm{R}} \circ 0_{\mathrm{R}}\right)
\end{gathered}
$$


It's easy to see that $U_{0}^{U},\left(\cdot, O_{\mathrm{R}}\right)$ and $\left\langle U_{0}^{U},\left(\circ, I_{\mathrm{R}}\right)\right\rangle$ are abelian groups. Moreover, $\left\langle U_{0}^{U} \cup\left\{0_{\mathrm{R}}\right\},\left(\cap, \bullet, \circ,^{-1}, \subseteq, 0_{\mathrm{R}}, O_{\mathrm{R}}, I_{\mathrm{R}}\right)\right\rangle$ is a bounded below compatible algebraic structure and $\left\langle U_{0}^{U} \cup\left\{1_{\mathrm{R}}\right\},\left(\cup, \bullet, \circ,{ }^{-1}, \subseteq, 1_{\mathrm{R}}, O_{\mathrm{R}}, I_{\mathrm{R}}\right)\right\rangle$ is a bounded above compatible algebraic structure.

Let's give other examples.

Let $F_{1} \subseteq U_{+1}^{U}$ be a set of partial and total functions are listed as $O_{\mathrm{R}}=\left\{\left(u_{1}, u_{2}\right),\left(u_{2}, u_{1}\right)\right\}$, $I_{\mathrm{R}}=\left\{\left(u_{1}, u_{1}\right),\left(u_{2}, u_{2}\right)\right\}, 0_{\mathrm{R}}=\varnothing, f_{1}=\left\{\left(u_{1}, u_{1}\right)\right\}, f_{2}=\left\{\left(u_{1}, u_{2}\right)\right\}, f_{3}=\left\{\left(u_{2}, u_{1}\right)\right\}, f_{4}=\left\{\left(u_{2}, u_{3}\right)\right\}$.

Cayley tables 4 and 5 describes the dual operations on the $F_{1}$.

Table 4. A Cayley table for $\circ$ on the set $F_{1}$.

\begin{tabular}{cccccccc}
\hline$\circ$ & $O_{\mathrm{R}}$ & $I_{\mathrm{R}}$ & $0_{\mathrm{R}}$ & $f_{1}$ & $f_{2}$ & $f_{3}$ & $f_{4}$ \\
\hline$O_{\mathrm{R}}$ & $I_{\mathrm{R}}$ & $O_{\mathrm{R}}$ & $0_{\mathrm{R}}$ & $f_{3}$ & $f_{4}$ & $f_{1}$ & $f_{2}$ \\
$I_{\mathrm{R}}$ & $O_{\mathrm{R}}$ & $I_{\mathrm{R}}$ & $0_{\mathrm{R}}$ & $f_{1}$ & $f_{2}$ & $f_{3}$ & $f_{4}$ \\
$0_{\mathrm{R}}$ & $0_{\mathrm{R}}$ & $0_{\mathrm{R}}$ & $0_{\mathrm{R}}$ & $0_{\mathrm{R}}$ & $0_{\mathrm{R}}$ & $0_{\mathrm{R}}$ & $0_{\mathrm{R}}$ \\
$f_{1}$ & $f_{2}$ & $f_{1}$ & $0_{\mathrm{R}}$ & $f_{1}$ & $f_{2}$ & $0_{\mathrm{R}}$ & $0_{\mathrm{R}}$ \\
$f_{2}$ & $f_{1}$ & $f_{2}$ & $0_{\mathrm{R}}$ & $0_{\mathrm{R}}$ & $0_{\mathrm{R}}$ & $f_{1}$ & $f_{2}$ \\
$f_{3}$ & $f_{4}$ & $f_{3}$ & $0_{\mathrm{R}}$ & $f_{3}$ & $f_{4}$ & $0_{\mathrm{R}}$ & $0_{\mathrm{R}}$ \\
$f_{4}$ & $f_{3}$ & $f_{4}$ & $0_{\mathrm{R}}$ & $0_{\mathrm{R}}$ & $0_{\mathrm{R}}$ & $f_{3}$ & $f_{4}$
\end{tabular}

Table 5. A Cayley table for - on the set $F_{1}$.

\begin{tabular}{cccccccc}
\hline$\cdot$ & $O_{\mathrm{R}}$ & $I_{\mathrm{R}}$ & $0_{\mathrm{R}}$ & $f_{1}$ & $f_{2}$ & $f_{3}$ & $f_{4}$ \\
\hline$O_{\mathrm{R}}$ & $O_{\mathrm{R}}$ & $I_{\mathrm{R}}$ & $0_{\mathrm{R}}$ & $f_{1}$ & $f_{2}$ & $f_{3}$ & $f_{4}$ \\
$I_{\mathrm{R}}$ & $I_{\mathrm{R}}$ & $O_{\mathrm{R}}$ & $0_{\mathrm{R}}$ & $f_{3}$ & $f_{4}$ & $f_{1}$ & $f_{2}$ \\
$0_{\mathrm{R}}$ & $0_{\mathrm{R}}$ & $0_{\mathrm{R}}$ & $0_{\mathrm{R}}$ & $0_{\mathrm{R}}$ & $0_{\mathrm{R}}$ & $0_{\mathrm{R}}$ & $0_{\mathrm{R}}$ \\
$f_{1}$ & $f_{1}$ & $f_{2}$ & $0_{\mathrm{R}}$ & $0_{\mathrm{R}}$ & $0_{\mathrm{R}}$ & $f_{1}$ & $f_{2}$ \\
$f_{2}$ & $f_{2}$ & $f_{1}$ & $0_{\mathrm{R}}$ & $f_{1}$ & $f_{2}$ & $0_{\mathrm{R}}$ & $0_{\mathrm{R}}$ \\
$f_{3}$ & $f_{3}$ & $f_{4}$ & $0_{\mathrm{R}}$ & $0_{\mathrm{R}}$ & $0_{\mathrm{R}}$ & $f_{3}$ & $f_{4}$ \\
$f_{4}$ & $f_{4}$ & $f_{3}$ & $0_{\mathrm{R}}$ & $f_{3}$ & $f_{4}$ & $0_{\mathrm{R}}$ & $0_{\mathrm{R}}$
\end{tabular}

It's easy to see that $\left\langle F_{1},\left(\cap, \bullet, \circ,{ }^{-1}, \subseteq, 0_{\mathrm{R}}, O_{\mathrm{R}}, I_{\mathrm{R}}\right)\right\rangle$ is a bounded below compatible algebraic structure.

Now let $F_{2} \subseteq U^{U}$ be a set of total functions are listed as $O_{\mathrm{R}}, I_{\mathrm{R}}, g_{1}=\left\{\left(u_{1}, u_{1}\right),\left(u_{2}, u_{1}\right)\right\}$, $g_{2}=\left\{\left(u_{1}, u_{2}\right),\left(u_{2}, u_{2}\right)\right\}$.

Cayley tables 6 and 7 describes the dual operations on the $F_{2}$.

Table 6. A Cayley table for $\circ$ on the set $F_{2}$.

\begin{tabular}{ccccc}
\hline$\circ$ & $O_{\mathrm{R}}$ & $I_{\mathrm{R}}$ & $g_{1}$ & $g_{2}$ \\
\hline$O_{\mathrm{R}}$ & $I_{\mathrm{R}}$ & $O_{\mathrm{R}}$ & $g_{1}$ & $g_{2}$ \\
$I_{\mathrm{R}}$ & $O_{\mathrm{R}}$ & $I_{\mathrm{R}}$ & $g_{1}$ & $g_{2}$ \\
$g_{1}$ & & $g_{1}$ & $g_{1}$ & $g_{2}$ \\
& $g_{1}$ & & $g_{1}$ & $g_{2}$
\end{tabular}


Table 7. A Cayley table for $\bullet$ on the set $F_{2}$.

\begin{tabular}{ccccc}
\hline$\bullet$ & $O_{\mathrm{R}}$ & $I_{\mathrm{R}}$ & $g_{1}$ & $g_{2}$ \\
\hline$O_{\mathrm{R}}$ & $O_{\mathrm{R}}$ & $I_{\mathrm{R}}$ & $g_{1}$ & $g_{2}$ \\
$I_{\mathrm{R}}$ & $I_{\mathrm{R}}$ & $O_{\mathrm{R}}$ & $g_{1}$ & $g_{2}$ \\
$g_{1}$ & $g_{1}$ & $g_{2}$ & $g_{1}$ & $g_{2}$ \\
$g_{2}$ & $g_{2}$ & $g_{1}$ & $g_{1}$ & $g_{2}$
\end{tabular}

In this case $\left\langle F_{2},\left(\cap, \bullet,,^{-1}, \subseteq, O_{\mathrm{R}}, I_{\mathrm{R}}\right)\right\rangle$ is unbounded compatible algebraic structure. Taking into account (42)-(43) we can write

$$
\begin{aligned}
& R_{1} \circ\left(R_{2} \cdot R_{3}\right)=\left(R_{1} \circ R_{2}\right) \cdot R_{3} \Leftrightarrow \bar{R}_{1} \cdot\left(\bar{R}_{2} \circ \overline{R_{3}}\right)=\left(\overline{R_{1}} \cdot \overline{R_{2}}\right) \circ \overline{R_{3}} \\
& \left(R_{2} \cdot R_{3}\right) \circ R_{1}=R_{2} \cdot\left(R_{3} \circ R_{1}\right) \Leftrightarrow\left(\bar{R}_{2} \circ \bar{R}_{3}\right) \cdot \bar{R}_{1}=\bar{R}_{2} \circ\left(\bar{R}_{3} \cdot \bar{R}_{1}\right)
\end{aligned}
$$

Let's denote the sets of relations are complement of functions from $F_{1}$ and $F_{2}$ as $\overline{F_{1}}=\left\{O_{\mathrm{R}}, I_{\mathrm{R}}, 1_{\mathrm{R}}, \overline{f_{1}}, \overline{f_{2}}, \overline{f_{3}}, \overline{f_{4}}\right\}$ and $\overline{F_{2}}=\left\{O_{\mathrm{R}}, I_{\mathrm{R}}, \overline{g_{1}}, \overline{g_{2}}\right\}$. In accordance with (71)-(72) we get ordered (not bounded and not lattice) compatible algebraic structures $\left\langle\bar{F}_{1},\left(\cup, \bullet, \circ,{ }^{-1}, \subseteq, 1_{\mathrm{R}}, O_{\mathrm{R}}, I_{\mathrm{R}}\right)\right\rangle$ and $\left\langle\overline{F_{2}},\left(\cdot, \circ, \subseteq, O_{\mathrm{R}}, I_{\mathrm{R}}\right)\right\rangle$.

Of cause, the list of examples can be continued.

\section{Conclusion}

We have studied non-traditional algebraic structures on the underlying set of binary relations. Starting from left composition, inclusion and Boolean isomorphism we defined dual ordered semigroups. Then we extended them to the more general ordered algebraic structure with a couple of dual operations. We have proved that these operations satisfy the semi-compatibility laws. This is notable and important fact. We paid special attention to the algebraic substructures satisfying the compatibility laws. So we have considered interesting examples of compatible algebraic structures.

The results will be useful for graphs and automatons as well as for coding, programming and artificial intelligence.

\section{References}

[1] Clifford A H and Preston G B 1961 The Algebraic Theory of Semigroups Mathematical Surveys and Monographs 7(1)

[2] Clifford A H and Preston G B 1967 The Algebraic Theory of Semigroups Mathematical Surveys and Monographs 7(2)

[3] Birkhoff G 1967 Lattice Theory (Providence RI: American Mathematical Society)

[4] Fuchs L 1963 Partially Ordered Algebraic Systems (Oxford: Pergamon Press)

[5] Ershov A P 1982 Abstract computability in algebraic systems Proc. Int. Symp. Algorithms in Modern Mathematics and its Applications (Novosibirsk: Computing Center of the Siberian Branch of the USSR Academy of Sciences) 2 194-299

[6] Chernov V M 2015 Quasiparallel algorithm for error-free convolution computation using reduced Mersenne-Lucas codes Computer Optics 39(2) 241-248 DOI: $10.18287 /$ 0134-2452-2015-39-2-241-248

[7] Chernov V M 2018 Calculation of Fourier-Galois transforms in reduced binary number systems Computer Optics 42(3) 495-500 DOI: 10.18287/ 0134-2452-2018-42-3-495-500

[8] Makhortov S D and Shurlin M D 2013 LP-Structures analysis: substantiation of refactoring in object-oriented programming Automation and Remote Control 74(7) 1211-1217 